INPLASY

PROTOCOL

To cite: Xue et al. Efficacy and Safety of Tonic Traditional Chinese Medicine in the Treatment of Depression : A Meta-Analysis and Data Mining. Inplasy protocol 202190068. doi: 10.37766/inplasy2021.9.0068

Received: 22 September 2021

Published: 22 September 2021

Corresponding author: Aisong Zhu

liaoningzhongyi@hotmail.com

Author Affiliation:

College of Basic Medical

Science, Zhejiang Chinese

Medical University.

Support: NSFC; NK R\&D PC.

Review Stage at time of this submission: Preliminary searches.

Conflicts of interest: None declared.

\section{Efficacy and Safety of Tonic Traditional Chinese Medicine in the Treatment of Depression: A Meta-Analysis and Data Mining}

Xue, D1; Song, Z2; Zhang, Y3; Jie, X4; Zhu, A5.

Review question / Objective: To evaluate the clinical effect of Tonic Chinese herbal medicine as adjuvant and alternative therapy in the treatment of depression. Comparing the adverse side effects of Tonic Chinese herbal medicine treatment on depression.

Condition being studied: Depression, characterized by more than two weeks of low mood, lack of energy, and loss of interest, is a chronic recurrent disorder. The World Health Organization (WHO) estimates that major depression (MDD) will rank the first cause of burden of disease by 2030. At present, the main treatment drugs are selective serotonin reuptake inhibitors (such as Fluoxetine). However, these drugs often take about 2-3 weeks to be effective. And long-term oral administration will be accompanied by a variety of adverse side reactions. Therefore, it is an urgent problem to be solved in modern clinical practice. Many studies have confirmed the efficacy of Traditional Chinese Medicine (TCM) in the treatment of depression. However, due to the large number of Chinese herbs, how to choose the effective herbs is a challenge. Under the guidance of Chinese medicine theory, we innovatively propose a research idea, that is according to the efficacy category of Chinese medicine to screen. This method is conducive to select the most effective herb. Tonic TCM herb is an important category of TCM and is widely used in the treatment of depression. However, there is no systematic review and meta-analysis of the treatment of depression with Tonic TCM.

INPLASY registration number: This protocol was registered with the International Platform of Registered Systematic Review and Meta-Analysis Protocols (INPLASY) on 22 September 2021 and was last updated on 22 September 2021 (registration number INPLASY202190068).

\section{INTRODUCTION}

Review question / Objective: To evaluate the clinical effect of Tonic Chinese herbal medicine as adjuvant and alternative therapy in the treatment of depression. Comparing the adverse side effects of 
Tonic Chinese herbal medicine treatment on depression.

Condition being studied: Depression, characterized by more than two weeks of low mood, lack of energy, and loss of interest, is a chronic recurrent disorder. The World Health Organization (WHO) estimates that major depression (MDD) will rank the first cause of burden of disease by 2030. At present, the main treatment drugs are selective serotonin reuptake inhibitors (such as Fluoxetine). However, these drugs often take about 2-3 weeks to be effective. And long-term oral administration will be accompanied by a variety of adverse side reactions. Therefore, it is an urgent problem to be solved in modern clinical practice. Many studies have confirmed the efficacy of Traditional Chinese Medicine

(TCM) in the treatment of depression. However, due to the large number of Chinese herbs, how to choose the effective herbs is a challenge. Under the guidance of Chinese medicine theory, we innovatively propose a research idea, that is according to the efficacy category of Chinese medicine to screen. This method is conducive to select the most effective herb. Tonic TCM herb is an important category of TCM and is widely used in the treatment of depression. However, there is no systematic review and meta-analysis of the treatment of depression with Tonic TCM.

\section{METHODS}

Search strategy: We will search the following electronic databases: PubMed, MEDLINE, EMBASE, The Cochrane Library, China National Knowledge Infrastructure (CNKI), Wanfang, Chinese Technology Journal Full-text Database (VIP) and China biomedical literature database (CBM) from their inception to September 1st, 2021. Various combinations of Medical Subject Headings and non-MeSH terms will be used based on the PICOS strategy (participants, interventions, comparison, results and studies). To achieve a comprehensive search, we will search all the literatures of TCM on depression and extract the research treated by Tonic TCM prescriptions. In addition, the references of initially included literature will be traced back to achieve a comprehensive search. And we will also search clinical registration websites for the ongoing trials on these sites. English and Chinese language will be included. We will include randomized controlled trials (RCTs).

Participant or population: Participants were adults with a primary diagnosis of MDD according to DSM-III, DSM-IV, DSM-IV-TR orICD-9, ICD-10(both in acute phase or in remission), regardless of gender, age, and ethnic group.

Intervention: The patients in the treatment groups will be given Tonic TCM interventions (regardless of the composition of the prescription, as long as it reflects the Therapy). Furthermore, trials including any other Chinese medicine therapy (e.g. soothing the liver, activating blood, etc.) and psychological therapies will be excluded.

Comparator: Patients in the control group will be given conventional treatment such as Fluoxetine, Escitalopram, etc.

Study designs to be included: We will include randomised trials to assess the beneficial effects of the treatments.

Eligibility criteria: Participants were adults with a primary diagnosis of MDD according to DSM-III, DSM-IV, DSM-IV-TR orICD-9, ICD-10(both in acute phase or in remission), regardless of gender, age, and ethnic group.

Information sources: We will search the following electronic databases: PubMed, MEDLINE, EMBASE, The Cochrane Library, China National Knowledge Infrastructure (CNKI), Wanfang, Chinese Technology Journal Full-text Database (VIP) and China biomedical literature database (CBM) from their inception to September 1st, 2021.Various combinations of Medical Subject Headings and non-MeSH terms will be used based on the PICOS strategy (participants, interventions, comparison, 
results and studies). To achieve a comprehensive search, we will search all the literatures of TCM on depression and extract the research treated by Tonic TCM prescriptions. In addition, the references of initially included literature will be traced back to achieve a comprehensive search. And we will also search clinical registration websites for the ongoing trials on these sites. English and Chinese language will be included. We will include randomized controlled trials (RCTs). Two authors will screen the titles and abstracts of the all records retrieved in above electronic databases independently to find potentially eligible reviews. According to the inclusion and exclusion criteria outlined above, the full texts of them will be retrieved for further identification. Any disagreement will be resolved by discussion or by consultation with a third author. The following data will be extracted:title, first author, year of publication, country of conduct, number of study arms, intervention, comparison, outcome, setting, and study design.

Main outcome(s): 1. Total effective rate; 2.Severity of depression. Change in depression score from baseline to the last available follow-up, measured using the Hamilton Depression Scale (HAMD); 3. TCM symptom scores which measured by Scale of TCM Syndrome Elements for Depression; 4. Adverse events.

Quality assessment / Risk of bias analysis: All authors will have an advanced training and use Cochrane Risk of Bias tool for the tool of quality assessment. Each article is assessed independently by two authors, When the two authors disagreed, discussion and ask for the third one. This tool assesses bias using seven items covering six different bias domains for each included study. The bias domains and items are: selection bias (random sequence generation and allocation concealment), performance bias (blinding of participants and personnel), detection bias (blinding of outcome assessment), attrition bias (incomplete outcome data), reporting bias (selective reporting), and other biases (other sources of bias). Each domain is assigned a judgment regarding the risk of bias within the included study for that domain using the labels of 'low risk' of bias, 'high risk' of bias, or 'unclear' risk of bias.

Strategy of data synthesis: We will use the Cochrane Collaboration Review Manager Software (RevMan, version 5.3) and STATA, version 11.0 for meta-analysis and statistical analyses. The heterogeneity in the included research results is judged by $I^{2}$ test. when there is no heterogeneity, the fixed effects model will be used for Metaanalysis. If not, use random effects model for analysis. Continuous variables is combined with mean difference (MD, or weighted mean difference, WMD) or standardized mean difference (SMD), and a $95 \%$ Cl confidence interval represented each effect size. Binomial variables will be expressed as odds ratio (OR).

Subgroup analysis: Due to SSRIs often take about 2-3 weeks to be effective, we will carry out subgroup analysis by treatment time. Besides, If the necessary, we also consider to conduct subgroup analysis based on the different intervention group and different level of depression severity.

Sensitivity analysis: Sensitivity analysis was performed to evaluate the robustness of the results. Publication bias was examined by visual inspection of funnel plots for asymmetry and Egger's test. The difference of $p \leq 0.05$ in Meta analysis was considered statistically significant.

\section{Country(ies) involved: China.}

Keywords: Depression; Tonic Traditional Chinese Medicine; Meta analysis; Data Mining.

Contributions of each author:

Author 1 - Dan Xue.

Email: 13295896226@163.com

Author 2 - Zhujin Song.

Author 3 - Yuhui Zhang.

Author 4 - Xiao Jie.

Author 5 - Aisong Zhu.

Email: liaoningzhongyi@hotmail.com 